\title{
QoS-Aware MAP Selection Scheme Based on Average Handover Delay for Multimedia Services in Multi-level HMIPv6 Networks
}

\author{
Y.-X. Lei and Z.-M. Zeng \\ Beijing University of Posts and Telecommunications \\ leiyixuebupt@hotmail.com
}

\begin{abstract}
In multi-level HMIPv6 (M-HMIPv6) networks the MN has to select a proper MAP using MAP selection schemes when migrating to the visited ARs. Currently, distance-based MAP Selection (DMS), Mobility-based MAP Selection (MMS) and Adaptive MAP Selection (AMS) schemes have been proposed, and AMS outperforms the other ones in several aspects. However, none of these schemes consider the handover Quality-of-Service (QoS) of mobile multimedia services as the metric for MAP selection. In this paper, we propose a novel QoS-aware MAP selection (QMS) scheme. In this scheme, the optimal MAP is determined according to the average handover delay (AHD) which characterizes the handover QoS for mobile multimedia services. We derive a lemma through theoretical analysis to estimate the AHD and design the procedure of QMS scheme based on this lemma. Through extensive NS-2 simulations, we verify the effectiveness of the proposed scheme and its advantage in AHD than AMS.
\end{abstract}

Keywords: Hierarchical Mobile IPv6 (HMIPv6), Mobility Anchor Point (MAP), handover delay, Quality of Service (QoS).

\section{Introduction}

Due to the limitation of Mobile IPv6 [1] for micro mobility management, Hierarchical Mobile IPv6 (HMIPv6) [2] [3] [10] was proposed by IETF to reduce the signaling cost and handover delay by adopting a Mobility Anchor Point (MAP) which can handle the localized mobility [3]. But in HMIPv6 networks with single MAP, the capacity and reliability of the networks need to be improved [5] [6] [10]. To meet this requirement, Multi-level HMIPv6 (M-HMIPv6) networks [5-9] were proposed inside which there are multiple MAPs. And, these MAPs forms different levels according to the number of hops from them to the Access Router (AR). In such circumstances, there exist more than one MAPs above the ARs, and it is essential for the MN to select an optimal MAP when it visits the ARs using MAP selection schemes. Several schemes have been proposed for MAP selection in M-HMIPv6 networks such as Distance-based MAP Selection (DMS) [2], Mobility-based MAP Selection (MMS) [8] [9], Adaptive MAP Selection (AMS) [6], mobile history-based MAP selection [12] and abstraction node based MAP selection schemes [7], etc. 
All the existing MAP selection schemes adopt different metrics including distance, mobile history, load balance, location update cost and packet delivery cost, etc. However, they don't consider QoS of mobile multimedia services, such as handover delay, as the metric for MAP selection [16]. We believe that handover QoS of mobile multimedia services are crucial issues that should be considered during MAP selection because the handover performance is strongly dependant upon the selection of MAPs.

In this paper, we propose a novel QoS-aware MAP selection scheme (QMS) which determines the optimal MAP based on average handover delay (AHD). We analyze the handover process theoretically, and derive a lemma to characterize the impact of MAP selection on AHD. Then, we conduct NS-2 simulations to verify our design. The results show that the analyses and the simulations are consistent. Finally, we compare our scheme with AMS, which is the most convincing scheme in the literature. The results show our scheme outperforms AMS in AHD.

The rest of the paper is organized as follows. Section 2 reviews the architecture of M-HMIPv6 networks, and gives a lemma to estimate the AHD in M-HMIPv6 networks which is the key design for QMS scheme. Then, the procedure of QMS scheme is given. In Section 3, numerical analyses and NS-2 simulations are conducted to verify our design. In Section 4, the performance of QMS and AMS is compared through NS-2 simulations. And, the conclusions are given in Section 5.

\section{QMS Scheme}

\subsection{Handover Process Analyses}

Fig. 1 shows the architecture of M-HMIPv6 networks. We can observe that there are multiple MAPs locating at different levels according to the number of hops from them
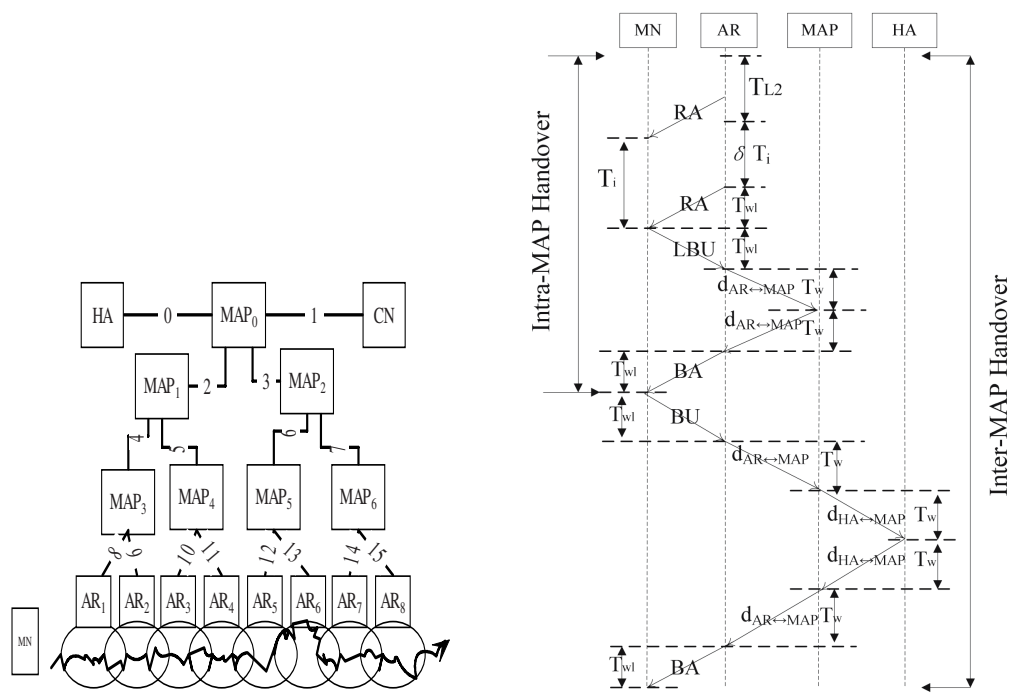

Fig. 1. An M-HMIPv6 network

Fig. 2. Inter-MAP and Intra-MAP Handover Procedure 
to the ARs. For example, $\mathrm{MAP}_{0}$ is the Level-1 MAP and there are three hops from it to an $\mathrm{AR}$, e.g., $\mathrm{AR}_{1}$. $\mathrm{MAP}_{1}$ and $\mathrm{MAP}_{2}$ are Level-2 MAPs since there are two hops away from $\mathrm{AR}_{1}$. To select a proper MAP for the MN, dynamic MAP discovery process [3] is conducted periodically. With this process, the information of available MAPs is collected and delivered to the ARs. When the MN migrates to an AR, e.g., $\mathrm{AR}_{2}$, it receives the Router Advertisement (RA) [1] [2] messages which contain the MAP options regarding to $\mathrm{MAP}_{0}, \mathrm{MAP}_{1}$ and $\mathrm{MAP}_{3}$. Then, an MN selects an MAP with certain MAP selection schemes according to the information within these MAP options. The key design of QMS scheme is Lemma I, which gives a lemma for estimating the AHD. The AHD is determined by two types of handovers, i.e., interMAP handover and intra-MAP handover which are shown in Fig. 2. Some abbreviations and definitions are listed in Table 1 to facilitate the descriptions in the following parts of the paper.

Table 1. ABBREVIATION AND DEFINITIONS

\begin{tabular}{|l|l|}
\hline$C N$ & Corespondent Node \\
\hline$(L) B U$ & (Local) Binding Update \\
\hline$B A$ & Acknowledgement for the BU \\
\hline$T_{L 2}$ & layer two handover delay \\
\hline$P D F$ & Probability Density Function \\
\hline$T_{i}$ & $\begin{array}{l}\text { the interval of Router Advertisement } \\
\text { of the ARs }\end{array}$ \\
\hline$d_{A \leftrightarrow B}$ & $\begin{array}{l}\text { The number of hops in wired link } \\
\text { connecting node A and B }\end{array}$ \\
\hline$T_{w l}$ & the transmission delay in wireless link \\
\hline$T_{w}$ & $\begin{array}{l}\text { the one hop transmission delay in } \\
\text { wired link }\end{array}$ \\
\hline$p_{a}$ & $\begin{array}{l}\text { the probability of MN to perform } \\
\text { intra-MAP handover }\end{array}$ \\
\hline$p_{m}$ & the probability of MN to perform \\
\hline
\end{tabular}

\begin{tabular}{|l|l|}
\hline & inter-MAP handover \\
\hline$d_{1}$ & intra-MAP handover delay \\
\hline$d_{2}$ & inter-MAP handover delay \\
\hline$\delta$ & $\begin{array}{l}\text { a positive variable between } 0 \text { and } 1 \\
\text { which follows uniform distribution }\end{array}$ \\
\hline$t_{s}$ & inter session time of the MN \\
\hline$t_{A}$ & AR residence time \\
\hline$t_{M}$ & MAP residence time \\
\hline$\lambda_{S}$ & session arrival rate \\
\hline$k_{A}$ & $\begin{array}{l}\text { The shape parameter of probability } \\
\text { density function (PDF) for } t_{A}\end{array}$ \\
\hline$\lambda_{A}$ & AR crossing rate \\
\hline$k_{M}$ & The shape parameter of PDF for $t_{M}$ \\
\hline$\lambda_{M}$ & MAP crossing rate \\
\hline
\end{tabular}

\subsection{The Lemma for AHD Estimation}

Lemma I: Given an M-HMIPv6 network like the one shown in Fig. 1 and handover process in Fig. 2, assume (1) the session arrival of the MN follows Poisson distribution, (2) the AR residence time and MAP residence time follow Gamma distribution with the parameters listed in Table 1, the AHDs of the MAP can be estimated as (1). In (1), $\rho$ stands for the ratio of $\lambda_{s}$ and $\lambda_{A}$ which is coined SessionMobility-Ratio (SMR) [7], and $n$ is the number of ARs covered by an MAP.

$$
A H D=\left(\frac{k_{A}}{K_{A}+\rho}\right)^{k_{A}}\left(T_{L 2}+3 T_{w l}+\frac{1}{2} T_{i}+2 T_{w} d_{A R \leftrightarrow M A P}\right)+\left(\frac{k_{M}}{k_{M}+\rho \sqrt{n}}\right)^{k_{w}}\left(T_{L 2}+5 T_{w l}+\frac{1}{2} T_{i}+4 T_{w} d_{A R \leftrightarrow M A P}+2 T_{w} d_{M A P \leftrightarrow H A}\right)
$$

We now prove Lemma I as follows. The PDFs of $t_{A}$ and $t_{M}$ are given in (2) and (3) respectively.

$$
f_{t_{A}}(t)=\frac{\left(k_{A} \lambda_{A}\right)^{k_{A}} t^{k_{A}-1}}{\Gamma\left(k_{A}\right)} e^{-k_{A} \lambda_{A} t}
$$




$$
f_{t_{M}}(t)=\frac{\left(k_{M} \lambda_{M}\right)^{k_{M}} t^{k_{M}-1}}{\Gamma\left(k_{M}\right)} e^{-k_{M} \lambda_{M} t}
$$

According to Fig. 2, we derive $d_{l}$ and $d_{2}$ as follows:

$$
\begin{gathered}
d_{1}=T_{L 2}+3 T_{w l}+\delta T_{i}+2 T_{w} d_{A R \leftrightarrow M A P} \\
d_{2}=T_{L 2}+5 T_{w l}+\delta T_{i}+4 T_{w} d_{A R \leftrightarrow M A P}+2 T_{w} d_{M A P \leftrightarrow H A}
\end{gathered}
$$

According to (4) and (5), we obtain AHD as (6).

$$
A H D=E\left(p_{a} d_{1}+p_{m} d_{2}\right)
$$

In (6), we can assume that $\delta$ is independent with $p_{a}$ and $p_{m}$, then we rewrite (6) as (7).

$$
A H D=p_{a}\left(T_{L 2}+3 T_{w l}+\frac{1}{2} T_{i}+2 T_{w} d_{A R \leftrightarrow M A P}\right)+p_{m}\left(T_{L 2}+5 T_{w l}+\frac{1}{2} T_{i}+4 T_{w} d_{A R \leftrightarrow M A P}+2 T_{w} d_{M A P \leftrightarrow H A}\right)
$$

According to (2) and (3), we deduce $p_{a}$ in (8).

$$
\begin{aligned}
& P_{a}=\int_{0}^{\infty} P\left(t>t_{A}\right) f_{t_{A}}(t) d t=\int_{0}^{\infty} e^{-\lambda_{s} t} \frac{\left(k_{A} \lambda_{M}\right)^{k_{A}} t^{k_{A}-1}}{\Gamma\left(k_{A}\right)} e^{-k_{A} \lambda_{M} t} d t \\
& =\left(\frac{k_{A} \lambda_{A}}{k_{A} \lambda_{A}+\lambda_{S}}\right)^{k_{A}} \int_{0}^{\infty} \frac{\left(k_{A} \lambda_{A}+\lambda_{s}\right)^{k_{A}} t^{k_{A}-1}}{\Gamma\left(k_{A}\right)} e^{-\left(k_{A} \lambda_{A}+\lambda_{s}\right) t} d t=\left(\frac{k_{A} \lambda_{A}}{K_{A} \lambda_{A}+\lambda_{S}}\right)^{k_{A}}=\left(\frac{k_{A}}{K_{A}+\frac{\lambda_{S}}{\lambda_{A}}}\right)^{k_{A}}
\end{aligned}
$$

Similarly, $p_{m}$ can be obtained as (9).

$$
P_{m}=\left(\frac{k_{M}}{k_{M}+\lambda_{s} / \lambda_{M}}\right)^{k_{M}}
$$

Then, take (8) and (9) into (7) and we get (10):

$$
A H D=\left(\frac{k_{A}}{K_{A}+\frac{\lambda_{s}}{\lambda_{A}}}\right)^{k_{A}}\left(T_{L 2}+3 T_{w}+\frac{1}{2} T_{i}+2 T_{w} d_{A R \leftrightarrow M A P}\right)+\left(\frac{k_{M}}{k_{M}+\frac{\lambda_{s}}{\lambda_{A}}}\right)^{k_{M}}\left(T_{L 2}+5 T_{w l}+\frac{1}{2} T_{i}+4 T_{w} d_{A R \leftrightarrow M A P}+2 T_{w} d_{M A P \leftrightarrow H A}\right)
$$

According to [6], we can assume $\lambda_{M}=\frac{\lambda_{A}}{\sqrt{n}}$ and $k_{M}=k_{A}$. Then (10) can be rewritten as (11).

$$
A H D=\left(\frac{k_{A}}{K_{A}+\rho}\right)^{k_{A}}\left(T_{L 2}+3 T_{w 1}+\frac{1}{2} T_{i}+2 T_{w} d_{A R \leftrightarrow M A P}\right)+\left(\frac{k_{M}}{k_{M}+\rho \sqrt{n}}\right)^{k_{k}}\left(T_{L 2}+5 T_{w 1}+\frac{1}{2} T_{i}+4 T_{w} d_{A R \leftrightarrow M A P}+2 T_{w} d_{M A P \leftrightarrow H A}\right)
$$

Up to now, we have proved Lemma I. Base on this lemma, we designed the procedure of QMS scheme as follows.

\subsection{Procedure of QMS Scheme}

The procedure of QMS scheme is as follows. (1) To obtain the MAP options. When an MN migrates to an AR, it obtains the MAPs above it from the MAP options within 
the RA messages. (2) To estimate SMR. The MN estimates SMR with the approach in [7]. (3) To extract the distances and the number of ARs an MAP covers. The MN obtains the hop number between AR and the available MAPs $\left(d_{A R \leftrightarrow M A P}\right)$, the distance from home agent (HA) to MAP $\left(d_{H A \leftrightarrow M A P}\right)$ and the number of ARs from the MAP option. To achieve this goal, the format of MAP option should be extended to contain these fields. The hops between HA and MAP can be obtained from routing protocols, and the number of ARs within one MAP can be obtained by configuration. (4) To estimate the AHD of each level MAP and select the optimal MAP. The AHDs of the MAPs in different levels are calculated according to Lemma I and the MAP which achieves the minimal AHD is selected by the MN. (5) Adaptive load control. If the optimal MAP is overloaded, then choose the suboptimal one until no MAP is available. In case of no available MAPs exist, the MN should register to the HA.

\section{Numerical Analyses and NS-2 Simulations for QMS Scheme}

In this section, we obtain some numerical results based on Lemma I, and conduct NS-2 simulations to verify the effectiveness of QMS scheme. The topology we used is shown in Fig. 1.

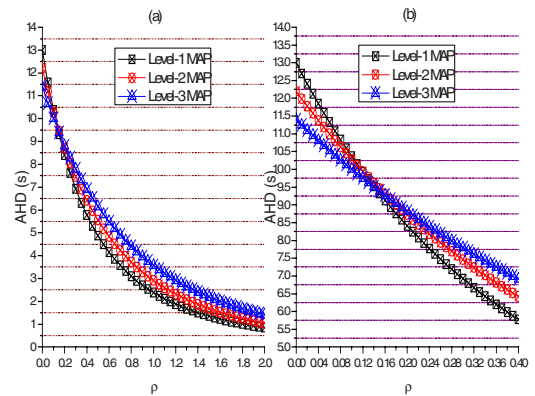

Fig. 3. AHD of different Levels of MAPs

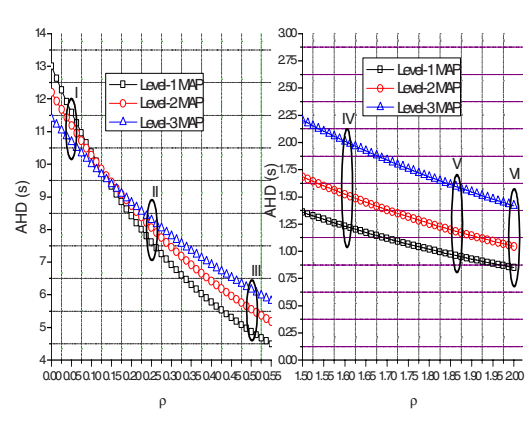

Fig. 4. AHD of Sampled $\rho$

\subsection{Numerical Results}

The parameters for numerical analysis are shown in Table 2.

Table 2. PARAMETERS FOR NUMERICAL ANALYSIS

\begin{tabular}{|l|l|l|l|l|l|l|}
\hline$T_{L 2}$ & $T_{w l}$ & $T_{w(\operatorname{Link} 2 \sim 15)}$ & $T_{w(\operatorname{Link} 0 \sim 1)}$ & $k_{A}\left(k_{M}\right)$ & $T_{i}$ & $d_{H A-M A P 0}$ \\
\hline $0.2 \mathrm{~s}$ & $0.5 \mathrm{~s}$ & $0.2 \mathrm{~s}$ & $2 \mathrm{~s}$ & 5 & $1 \mathrm{~s}$ & 10 \\
\hline
\end{tabular}

In (11), $d_{H A \leftrightarrow M A P}$ and $d_{A R \leftrightarrow M A P}$ are determined by which MAP is selected by the MN. We denote the mean MAP crossing rate of three levels of MAPs as $\lambda^{1}{ }_{M} \sim \lambda^{3}{ }_{M}$. According to [7], we obtain (12). 


$$
\lambda_{M}{ }^{1}=\frac{\lambda_{M}^{2}}{\sqrt{2}}=\frac{\lambda_{M}^{3}}{2}=\frac{\lambda_{A}}{2 \sqrt{2}}
$$

We plot AHD of different levels of MAPs in Fig. 3 (a) and (b). Fig. 3 (a) is the whole graph, and Fig. 3 (b) enlarges the part with $\rho$ ranging from 0 to 0.4 . We observe that the optimal AHD is obtained by different levels of MAPs with the growing of $\rho$. For example, when $\rho$ equals 0.0125, level-3 MAPs achieve the lowest AHD. And, when $\rho$ is 0.5 , level- 1 obtains the optimal AHD.

\subsection{NS-2 Simulations}

The simulation runs with NS-2.1b6a [15] installed in Redhat Linux 9.0. Robert Hsieh [4] previously contributed an extension to NS-2 concerning the Fast Handover for Hierarchical Mobile IPv6 (F-HMIPv6) [13] without implementing the MAP discovery and MAP selection functions in HMIPv6 protocol [2]. We have added the missed functions according the latest protocol standard [2], and then implement MAP selection schemes for performance evaluation. To verify the effectiveness of QMS, we adopt the same network topology which is used for numerical analysis. The coordinates of the 8 ARs are as follows: $(250,250),(375,250),(500,250),(625,250)$, $(250,375),(375,375),(500,375),(625,375)$. The radius of the circular area covered by each $\mathrm{AR}$ is $75 \mathrm{~m}$. According to the radius, we set the receive threshold as $3.41828 \mathrm{e}-8 \mathrm{w}(-49.466 \mathrm{dBm})$ and the transmission power of AR as $0.28183815 \mathrm{w}$. To approximate the numerical analysis in the previous section, we set the delay of the wired links as Table 2. And, the bandwidth of all the wired links is $100 \mathrm{Mbps}$.

We adopt CBR traffics based on UDP. The CBR traffic is unidirectional and originated from $\mathrm{CN}$ to $\mathrm{MN}$ with the bandwidth and packet size as $128 \mathrm{kbps}$ and 512 bytes respectively. We obtain the AHD from UDP traffic which is defined as the interval from the instant of receiving the last packet at previous AR to the instant of receiving the first packet at new AR. The call arrival is follows Poisson distribution and the mobility of the MN simulated by Random Waypoint model [12] generated by a Java-based scenario program [14]. The simulation time is 10 hours, and the results are obtained by averaging the statistics over 10 runs.

Table 3. SIX SCENARIOS FOR SIMULATIONS

\begin{tabular}{|l|l|l|l|}
\hline Scenario Index & \multicolumn{1}{|c|}{ speed range } & call arrival rate & samples of $\rho$ \\
\hline I & $40-60 \mathrm{~m} / \mathrm{s}$ & 0.0375 & 0.05 \\
\hline II & $40-60 \mathrm{~m} / \mathrm{s}$ & 0.1875 & 0.25 \\
\hline III & $5-15 \mathrm{~m} / \mathrm{s}$ & 1.8750 & 0.5 \\
\hline IV & $5-15 \mathrm{~m} / \mathrm{s}$ & 6.0 & 1.6 \\
\hline V & $5-15 \mathrm{~m} / \mathrm{s}$ & 6.9375 & 1.85 \\
\hline VI & $5-15 \mathrm{~m} / \mathrm{s}$ & 7.3125 & 2.00 \\
\hline
\end{tabular}

During the simulation, the MN chooses different level of MAPs in each scenario, shown in Table 3, and each scenario refers to a sample of $\rho$. The numerical results of the samples of $\rho$ are plotted in Fig. 4, and the simulation results of the samples of $\rho$ are provided in Fig. 5. From Fig. 4 and Fig. 5, we observe that the orders of AHDs incurred by different MAP level from numerical analysis are consistent with the orders in simulation results for all the scenarios. For example, when $\rho$ equals 0.50 , $\mathrm{AHD}_{1}<\mathrm{AHD}_{2}<\mathrm{AHD}_{3}$ holds in both simulations and analyses results. 


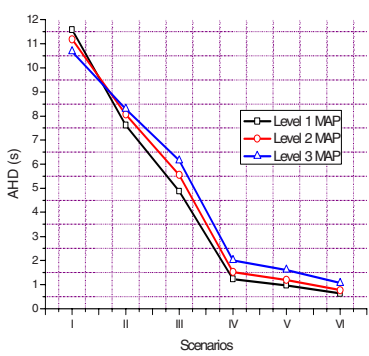

Fig. 5. AHD of Simulations

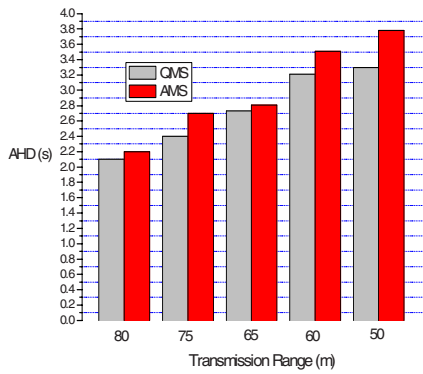

Fig. 6. Comparison of AHD between AMS and QMS

\section{Comparison with AMS Through NS-2 Simulations}

Since AMS [6] is the most convincing scheme in the literature, we compare the performance of QMS with AMS in AHD through NS-2 simulations. The topology and traffic scenarios are the same with that in Section 3.2.

We simulated $120 \mathrm{MNs}$ with different session arrival rates and mobility patterns. The capacity of the MAPs, i.e., the number of concurrent MNs that can be served by an MAP, is larger than the number of MNs we simulated. And the AHD in this section is the mean of handover delay for the sessions of all the MNs, not for only one $\mathrm{MN}$ as defined in previous sections. The simulation results are shown in Fig. 6. We change the transmission range of the ARs in different scenarios, and the results show that QMS can effectively reduce the AHD for all the senarios compared with AMS. The main reason is that it considers the handover delay directly as the criteria for MAP selection.

\section{Conclusion}

This paper proposes a novel QMS scheme in M-HMIPv6 networks. Firstly, we analyze the handover process theoretically and derive a lemma to estimate AHD, which is the key design of QMS scheme. Then, we verify the analyses by comparing the numerical results with the statistics from NS-2 simulations. Moreover, the performance of QMS is compared with AMS, which is considered as the most convincing MAP selection scheme in the literature. The results show that QMS outperforms AMS in AHD. The proposed scheme can be applied for handover optimization for multimedia services in M-HMIPv6 networks. In future works, we will extend the theoretical analyses to cover more generic mobility models, and conduct more comprehensive simulations to evaluate the performance of QMS scheme on other factors, such as location update cost and packet delivery cost. We will also study how to apply QMS scheme in F-HMIPv6 [13] networks to achieve better handover performance for multimedia services in M-HMIPv6 networks. 
Acknowledgments. This research is supported by the Education doctoral special fund, No. 20040013010.

\section{References}

1. D. Johnson, C. Perkins and J. Arkko: Mobility Support in IPv6, IETF RFC 3775, (June 2004)

2. H. Soliman, C. Castelluccia, K. El Malki and L. Bellier, "Hierarchical Mobile IPv6 Mobility Management," IETF RFC 4140, (August 2005)

3. M. H. Habaebi: Macro/micro-mobility Fast Handover in Hierarchical Mobile IPv6, Computer Communications, Vol. 29, (2006), 611-617

4. Robert Hsieh, Aruna Seneviratne, Hesham Soliman and Karim El-Malki: Performance Analysis on Hierarchical Mobile IPv6 with Fast-handoff over End-to-end TCP, Proc. of EEE Globecom 2002, Vol. 21, No. 1, (November 2002), 2500-2504

5. Kawano K., Kinoshita K. and Murakami K., Multilevel Hierarchical Mobility Management Scheme in Complicated Structured Networks, Proc. of 29th Annual IEEE International Conference on Local Computer Networks, (November 2004), 34-41

6. Sangheon Pack, Minji Nam, Taeyoung Kwon and Yanghee Choi, An Adaptive Mobility Anchor Point Selection Scheme in Hierarchical Mobile IPv6 Networks, Elsevier Computer Communications, Vol. 29, No. 16, (2006), 3065-3078

7. Takaashi Kumagai, Takuya Asaka and Tatsuro Takahashi: "Location Management Using Mobile History for Hierarchical Mobile IPv6 Networks," Proc. of IEEE Globecom 2004, Vol 3, (December 2004), 1585-1589

8. E. Natalizio, A. Scicchitano and S. Marano: Mobility Anchor Point Selection Based on User Mobility in HMIPv6 Integrated with Fast Handover Mechanism, Proc. of IEEE WCNC 2005, Vol. 3, (March 2005), 1434-1439

9. Taleb. T, Suzuki T., Nei Kato and Nemoto Y.: A Dynamic and Efficient MAP Selection Scheme for Mobile IPv6 Networks, Proc. of IEEE Globecom 2005, Vol. 5, (December 2005), 2891-2895

10. Youngjunce Gwon, James Kempf and Alper Yegin: Scalability and Robustness Analysis of Mobile IPv6, Fast Mobile IPv6, Hierarchical Mobile IPv6 and Hybrid IPv6 Mobility Protocols Using a Large-scale Simulation, Proc. of IEEE ICC 2004, Vol. 7, (June 2004), 4087-4091

11. Y.-W. Chen and M.-J. Huang: A Novel MAP Selection Scheme by Using Abstraction Node in Hierarchical MIPv6," to be pubished in Proc. of IEEE ICC 2006, (2006)

12. T. Camp, J. Boleng, and V. Davies: A Survey of Mobility Models for Ad Hoc Network Research, Wireless Communication \& Mobile Computing (WCMC): Special Issue on Mobile Ad Hoc Networking: Research, Trends and Applications, Vol. 2, No. 5, (2002), 483-502

13. HeeYoung Jung, Gesham Soliman, Seok Joo Koh and Jae Yong Lee: Fast Handover for Hierarchical MIPv6, IETF Internet Draft, (2005)

14. http://web.informatik.uni-bonn.de/IV/Mitarbeiter/dewaal/BonnMotion/

15. http://www.isi.edu/nsnam/ns

16. Yi-Xue Lei, et. al.: Impact of MAP Selection on Handover Performance for Multimedia Services in Multi-level HMIPv6 Networks, to be published in Proc. of IEEE WCNC 2007, (2007) 